\section{Limited number of patrilines in horse domestication}

Gabriella Lindgren $^{1}$, Niclas Backström ${ }^{1}$, June Swinburne ${ }^{2}$, Linda Hellborg ${ }^{1}$, Annika Einarsson ${ }^{1}$, Kaj Sandberg ${ }^{3}$, Gus Cothran ${ }^{4}$, Carles Vilà ${ }^{1}$, Matthew Binns ${ }^{2} \&$ Hans Ellegren ${ }^{1}$

Genetic studies using mitochondrial DNA (mtDNA) have identified extensive matrilinear diversity among domestic horses. Here, we show that this high degree of polymorphism is not matched by a corresponding patrilinear diversity of the malespecific $\mathrm{Y}$ chromosome. In fact, a screening for single-nucleotide polymorphisms (SNPs) in $\mathbf{1 4 . 3} \mathrm{kb}$ of noncoding Y chromosome sequence among 52 male horses of 15 different breeds did not identify a single segregation site. These observations are consistent with a strong sex-bias in the domestication process, with few stallions contributing genetically to the domestic horse.

The finding of $>90 \mathrm{mtDNA}$ haplotypes among domestic horses indicates the incorporation of numerous genetic lineages into the breeding stock $^{1-3}$. These data support domestication from geographically separate areas, which may have occurred through diffusion of the required human expertise or through truly independent domestication processes. But genetic diversity in mtDNA only reflects the maternal contribution to the gene pool. Recently, genetic markers from the male-specific Y chromosome have unveiled the patterns of evolution and migration among modern humans, as manifested by paternal genetic architectures ${ }^{4}$. Y-chromosome markers will also be informative for addressing the genetic and anthropological processes associated with animal domestication ${ }^{5}$, as males and females may have been treated differently by early human societies ${ }^{6}$.

To study the genetic contribution of stallions in horse domestication, we screened for SNPs in $14.3 \mathrm{~kb}$ of equine Y-chromosome sequence, divided into 37 fragments (Table 1 and Supplementary Methods online). Because many breeds may derive from a small number of founders or from a limited number of registries in the studbook, bottleneck and founder effects may have reduced the genetic diversity within each single breed. Consequently, we used 52 male horses from 15 different breeds for screening, including divergent European (Ardennais, Connemara, Exmoor, Fjord, Gotland, Icelandic, Shetland, NorthSwedish and Thoroughbred) and Asian (Akhal Teké, Arab, Caspian Pony, Khuzestan Arab, Malwari and Thai Pony) breeds. These breeds were selected to represent a wide variety of horses and ponies to cover as much as possible of the gene pool of the domestic horse; most of the breeds have old histories and substantial phenotypic variation.
Table 1 Description of horse Y-chromosome markers

\begin{tabular}{|c|c|c|c|c|}
\hline \multirow{2}{*}{ Locus } & \multirow{2}{*}{ Length (bp) } & \multicolumn{3}{|c|}{ GenBank accession number } \\
\hline & & $\begin{array}{l}\text { Equus } \\
\text { caballus }\end{array}$ & $\begin{array}{c}\text { Equus caballus } \\
\text { przewalskii }\end{array}$ & $\begin{array}{l}\text { Equus } \\
\text { asinus }\end{array}$ \\
\hline \multicolumn{5}{|c|}{ Y-chromosome introns } \\
\hline$A M E L Y 1$ & 394 & AB091794 & AY532824 & AY532815 \\
\hline AMELY2 & 358 & AB091794 & AY532825 & AY532816 \\
\hline AMELY3 & 481 & AB091794 & AY532826 & AY532817 \\
\hline AMELY4 & 488 & AB091794 & AY532827 & AY532818 \\
\hline AMELYG & 470 & AB091794 & AY532828 & AY532819 \\
\hline AMELY7 & 215 & AB091794 & AY532829 & AY532820 \\
\hline AMELY 8 & 478 & AB091794 & AY532830 & AY532821 \\
\hline AMELY9 & 203 & AB091794 & AY532831 & AY532822 \\
\hline AMELY11 & 245 & AB091794 & AY532832 & AY532823 \\
\hline$D B Y 7$ & 259 & AY532880 & AY532884 & AY532882 \\
\hline$D B Y 8$ & 85 & AY532881 & AY532885 & AY532883 \\
\hline SMCY2 & 251 & AY532886 & AY532894 & AY532890 \\
\hline SMCY3 & 848 & AY532887 & AY532895 & AY532891 \\
\hline SMCY7 & 341 & AY532888 & AY532896 & AY532892 \\
\hline SMCY17 & 80 & AY532889 & AY532897 & AY532893 \\
\hline$S R Y^{*}$ & 452 & AB004572 & AY532879 & AY532878 \\
\hline \multicolumn{5}{|c|}{ Subclones from $Z F Y$-positive BAC } \\
\hline$Z F Y D$ & 710 & AY532845 & AY532860 & AY532833 \\
\hline$Z F Y G$ & 539 & AY532846 & AY532861 & AY532834 \\
\hline ZFYH & 579 & AY532847 & AY532862 & AY532835 \\
\hline ZFY27A & 323 & AY532848 & AY532863 & AY532836 \\
\hline ZFY43A & 435 & AY532849 & AY532864 & AY532837 \\
\hline ZFY43B2 & 452 & AY532875 & AY532877 & AY532876 \\
\hline ZFY44A & 391 & AY532850 & AY532865 & ND \\
\hline ZFY46A & 341 & AY532851 & AY532866 & AY532838 \\
\hline ZFY5OA & 252 & AY532852 & AY532867 & ND \\
\hline ZFY5OB & 314 & AY532853 & AY532868 & ND \\
\hline ZFY51A & 353 & AY532854 & AY532869 & AY532839 \\
\hline ZFY52A & 381 & AY532855 & AY532870 & AY532840 \\
\hline ZFY53A & 414 & AY532856 & AY532871 & AY532841 \\
\hline ZFY53B & 358 & AY532857 & AY532872 & AY532842 \\
\hline ZFY55A & 342 & AY532858 & AY532873 & AY532843 \\
\hline ZFY55B & 426 & AY532859 & AY532874 & AY532844 \\
\hline \multicolumn{5}{|c|}{ Anonymous Y-linked fragments } \\
\hline Eca-Y2B17 & 438 & G72335 & AY532806 & AY532805 \\
\hline Eca-Y3B1 & 468 & G72336 & AY532811 & AY532807 \\
\hline Eca-Y3B8 & 445 & G72337 & AY532812 & AY532808 \\
\hline Eca-Y3B12 & 392 & G72338 & AY532813 & AY532809 \\
\hline Eca-Y3B19 & 215 & G72339 & AY532814 & AY532810 \\
\hline
\end{tabular}

All fragments were sequenced in 52 male horses from 15 divergent breeds. ND, not determined. *Untranslated region.

${ }^{1}$ Department of Evolutionary Biology, Evolutionary Biology Centre, Uppsala University, Norbyvägen 18D, SE-752 36 Uppsala, Sweden. ${ }^{2}$ Animal Health Trust, Newmarket, CB8 7UU, UK. ${ }^{3}$ Department of Animal Breeding and Genetics, Swedish University of Agricultural Sciences, Box 7023 , SE-750 07 Uppsala, Sweden. ${ }^{4}$ Department of Veterinary Science, University of Kentucky, Lexington, Kentucky 40546-0076, USA. Correspondence should be addressed to H.E.

(Hans.Ellegren@ebc.uu.se).

Published online 14 March 2004; doi:10.1038/ng1326 
All stallions carried the same Y-chromosome haplotype. This contrasts sharply with the extensive mtDNA diversity and indicates very low levels of Y-chromosome variability in domestic horses. Limited polymorphism is not a general feature of equine nuclear DNA, as a screening of $2.3 \mathrm{~kb}$ of X-chromosome intron sequence in a subset of the individuals identified 17 SNPs and a nucleotide diversity $(\pi)$ of 1.4 $\times 10^{-3} \pm 0.1 \times 10^{-3}$ (Supplementary Table 1 online). Nor is it due to selective constraints on the $\mathrm{Y}$ chromosome, because, by determining the orthologous $\mathrm{X}$ - and Y-chromosome sequence in donkey, we found strong statistical support for lower levels of variability on $\mathrm{Y}$ than on $\mathrm{X}$ (HKA test $P<0.00001$ ).

The ancestors to domestic horses have gone extinct in the wild, but a population of Mongolian wild horses, or Przewalski's horses (Equus caballus przewalskii), is kept in captivity. We sequenced one male Przewalski's horse for all Y-chromosome fragments and found that it differed from all domestic horses at six nucleotide positions over the $14.3 \mathrm{~kb}$ and also had a 7-bp deletion. Based on Y-chromosome data, the split between Przewalski's horse and domestic horses is estimated to have occurred $120,000-240,000$ years ago $^{7}$. As this is long before wild horses were domesticated $\left(\sim 6,000\right.$ years ago $\left.{ }^{8}\right)$, wild horses may have had Y-chromosome variability before domestication.

How low is Y-chromosome variability of domestic horses compared with that of, for instance, humans? Several large-scale studies uniformly estimated $\pi$ in noncoding sequences of the human Y chromosome at $1.0-1.5 \times 10^{-4}$ (refs. 9-12). One of these studies ${ }^{9}$ was similar to ours: 50 chromosomes from geographically diverse human samples were screened in $35.3 \mathrm{~kb}$ of SMCY intron sequence, identifying 37 segregating sites or one every $950 \mathrm{bp}$ (larger studies found one every 576-840 bp; refs. 9,11,12). A randomization test provided strong statistical support for a significant difference in Y-chromosome polymorphism levels between horses and humans $(P<0.0001)$. Quantification of this difference is not straightforward, as our domestic horse sample was monomorphic for $\mathrm{Y}$ chromosome sequences. But we can conservatively assess the difference by assigning a variable site to the horse data set. With one rare allele at $2 \%$ frequency, $\pi$ would be $3 \times 10^{-6}$; at $10 \%$ frequency, $\pi$ would be $1 \times 10^{-5}$. This implies that $\pi$ estimates for the $\mathrm{Y}$ chromosome are at least 10-30 times lower for domestic horses than for humans.

Our observations are compatible with a scenario of strong sex bias in breeding with only a limited number of sires contributing genetically to the domestic horse (low male effective population size). Modern breeding practice selects stallions and lets them cover many mares each, a breeding scheme that reduces the number of patrilines in the population $^{13}$. Our data suggest that using a limited number of stallions in breeding may be traditional breeding practice ${ }^{14}$ and thus date back to the initial phase of horse domestication. If a strong sex bias in breeding were only a modern, breed-specific phenomenon, we would expect to see some Y-chromosome variability among breeds. Moreover, a bias in the early exploitation of each sex could also have contributed to a limited male gene pool. During the Paleolithic age, horses were an important part of the human diet ${ }^{15}$, and this could have been the case during the early stages of horse domestication as well. In general, food production is maximized if most males are consumed and females are left for reproduction; such a sex bias in exploitation of several domestic animals is suggested from archaeological records ${ }^{6}$.

Our observations do not exclude the possibility that the equine $\mathrm{Y}$ chromosome was low in variability before the time of domestication. The social structure of the wild horses from which domestic breeds evolved probably featured a single stallion holding a harem of multiple mares, implying skewed reproductive success of males. As mtDNA data point at domestication from geographically widespread areas, however, divergent Y-chromosome lineages might have been incorporated into the breeding stock even if local Y-chromosome variability was low. An alternative scenario would imply a single domestication event in a restricted geographical region, resulting in the incorporation of only a limited number of Y-chromosome haplotypes into the breeding stock. When domestic animals, or pastoralism in itself, then spread from one locality to another, the maternal gene pool may have been diversified by the capture of only wild females from local populations (while backcrosses with wild stallions were prevented). Under this scenario, the contrasting levels of variability in mtDNA and the $\mathrm{Y}$ chromosome seen in modern horses reflect how the practice of horse domestication spread among early human societies.

Note: Supplementary information is available on the Nature Genetics website.

\section{ACKNOWLEDGMENTS}

We thank L. Skow for access to the BAC library; M. Webster, J. Seddon and A. Eriksson for help and discussion; and M. Oom, L. Firouz and A. Tawatsin for help with samples. Financial support was obtained from the Swedish Research Council for Environment, Agricultural Sciences and Spatial Planning. H.E. is a Royal Academy of Sciences Research fellow supported by a grant from the Knut and Alice Wallenberg foundation.

\section{COMPETING INTERESTS STATEMENT}

The authors declare that they have no competing financial interests.

Received 19 December 2003; accepted 12 February 2004

Published online at http://www.nature.com/naturegenetics/

1. Lister, A.M. et al. Ancient Biomol. 2, 267-280 (1998).

2. Vilá, C. et al. Science 291, 474-477 (2001).

3. Jansen, T. et al. Proc. Natl. Acad. Sci. USA 99, 10905-10910 (2002)

4. Goldstein, D.B. Science 291, 1738-1742 (2001).

5. MacHugh, D.E. \& Bradley, D.G. Proc. Natl. Acad. Sci. USA 98, 5382-5384 (2001).

6. Zeder, M.A. \& Hesse, B. Science 287, 2254-2257 (2000).

7. Wallner, B. et al. Anim. Genet. 34, 453-456 (2003).

8. Clutton-Brock, J. A Natural History of Domesticated Mammals. (Cambridge University Press, Cambridge, Massachusetts, 1999).

9. Shen, P. et al. Proc. Natl. Acad. Sci. USA 97, 7354-7359 (2000).

10. The International SNP Map Working Group. Nature 409, 928 (2001).

11. Hammer, M.F. et al. Mol. Biol. Evol. 18, 1189-1203 (2001).

12. Hammer, M.F. et al. Genetics 164, 1495-1509 (2003).

13. Cunningham, E.P. et al. Anim. Genet. 32, 360 (2001).

14. Levine, M.A. J. Anthropol. Archaeol. 18, 29-78 (1999).

15. Olsen, S.L. Horses Through Time. (Carnegie Museum of Natural History, Pittsburgh, Pennsylvania, 1996). 